CLINICAL STUDY

\title{
Connective tissue growth factor expression in endocrine tumors is associated with high stromal expression of $\alpha$-smooth muscle actin
}

\author{
Janet L Cunningham, Apostolos V Tsolakis, Annica Jacobson and Eva T Janson \\ Section of Endocrine Oncology, Department of Medical Sciences, Lab 14, Research Department 2, Uppsala University Hospital, Uppsala University, \\ SE 75185 Uppsala, Sweden \\ (Correspondence should be addressed to J L Cunningham; Email: janet.cunningham@medsci.uu.se)
}

\begin{abstract}
Objective: Complications due to fibrosis development are common in patients with well-differentiated endocrine carcinomas in the small intestine (ileal carcinoids). Connective tissue growth factor (CTGF) expression in ileal carcinoids may be related to this fibrosis development. This study aimed to examine CTGF expression in relation to local myofibroblast differentiation in a large series of ileal carcinoids and in different types of endocrine tumors.

Methods: Immunoreactivity (IR) for CTGF and $\alpha$-smooth muscle actin ( $\alpha$-SMA), a marker for myofibroblasts, was compared in serial tumor tissue sections from 42 patients with ileal carcinoids and from 80 patients with other endocrine tumors. Western blot was performed on an additional 21 patients with ileal carcinoids.

Results: CTGF IR was present in $>50 \%$ of tumor cells in all 42 ileal carcinoids and in 2 out of 14 endocrine pancreatic tumors, 4 out of 6 rectal carcinoids, and 1 out of 5 lung carcinoids. Tumors with abundant CTGF expression also displayed $\alpha$-SMA IR in stromal fibroblast-like cells, whereas other endocrine tumors displayed less or no CTGF and $\alpha$-SMA IR. Protein bands corresponding to full-length CTGF (36-42 kDa) were detected in protein lysates from ileal carcinoids.

Conclusion: CTGF is uniquely prevalent in ileal carcinoids when compared with most other endocrine tumor types. Immunoreactive cells are adjacent areas with increased fibrovascular stroma that express $\alpha$-SMA. This supports a potential role for CTGF in myofibroblast-mediated fibrosis associated with ileal carcinoids, and indicates that CTGF should be investigated as a target for future therapy.
\end{abstract}

European Journal of Endocrinology 163 691-697

\section{Introduction}

Well-differentiated endocrine carcinomas with serotonin production originating in the small intestine and proximal colon are derived from enterochromaffin cells and commonly denoted ileal carcinoids (1). These tumors are associated with fibrosis development within and surrounding the tumor. In the gut, fibrosis can cause intestinal obstruction, ischemia, and in rare cases, ureteral obstruction. Fibrotic complications are reported to arise in $16-48 \%$ of patients. The development of other fibrotic conditions in distant organs, such as carcinoid heart disease, supports the theory that fibrosis formation is stimulated by substances produced and secreted by the tumor. The mechanism behind carcinoid-associated fibrosis is not established; yet, the list of factors that have been implicated is long. Carcinoid-produced hormones, serotonin (2) and tachykinins (3-5), have been shown to be related to fibroblast growth and fibrotic changes in the heart valves. Growth factors produced in ileal carcinoids such as platelet-derived growth factor, insulin-like growth factors 1 and 2 (IGF1 and 2), epidermal growth factor (EGF), and transforming growth factors $\alpha$ and $\beta$ (TGFA and TGFB) and their receptors have also been postulated as potential mediators in the context of carcinoid fibrosis (6-9).

Connective tissue growth factor (CTGF/CCN2) is a 349-amino acid polypeptide, which is transcriptionally activated by TGFB (10). Furthermore, it has been shown to mediate some of the extracellular matrix-inducing properties that have been previously attributed to TGFB (11-13). CTGF is expressed in ileal carcinoids, and fulllength CTGF can be found in patient serum and is potentially involved in carcinoid-associated fibrotic disease (14).

TGFB has been shown to have multiple roles in many different fibrotic conditions $(15,16)$, and it is highly expressed in ileal carcinoids $(9,17)$. TGFB-induced fibroblast proliferation, collagen synthesis, and myofibroblast differentiation have been shown to be mediated by CTGF-dependent pathways $(11,18)$. CTGF has been 
studied in multiple contexts and attributed many functions. The results in different systems are in some ways contradictory. It is becoming apparent that CTGF function is highly dependent on its processing. CTGF stimulation of fibroblast proliferation and collagen synthesis are, for example, separate processes. In vitro experiments demonstrate that the $\mathrm{N}$-terminal region of CTGF stimulates myofibroblast differentiation and collagen production, while the C-terminal stimulates fibroblast cell proliferation (19). The presence of other GHs, such as EGF, IGF2, and TGFB, also seems to influence the degree to which these different fibrotic processes are active (18).

Our aim was to characterize CTGF expression in primary tumors and metastases from ileal carcinoids in relation to other endocrine tumor types by means of immunohistochemistry (IHC). Furthermore, we studied the possible relationship between CTGF and fibrosis in endocrine tumors by comparing the expression of CTGF and $\alpha$-smooth muscle actin ( $\alpha$-SMA), a marker for myofibroblast differentiation. We further characterized CTGF in primary tumors and metastases from ileal carcinoids using western immunoblotting.

\section{Materials and methods}

\section{Patients}

This study included 124 patients. The tumors were diagnosed histopathologically at the Laboratory of Pathology and Cytology, Uppsala University Hospital, Sweden. Tumors from 47 patients with ileal carcinoids displayed chromogranin A and serotonin immunoreactivity (IR), and the patients had elevated urinary excretion of 5-hydroxy imidazole acetic acid and had metastatic disease at diagnosis. The diagnosis of the other endocrine tumors was based on international recommendations for the classification of endocrine tumors $(20,21)$.

For IHC analysis, ileal carcinoid tumor material, consisting of 26 primary tumors, 21 mesentery metastases, and 13 liver metastases, from 42 patients was used. The clinical data for this study also included primary tumors from an additional 80 patients with various endocrine tumors: ECLomas type $1(n=5)$, ECLomas type $3(n=3)$, lung carcinoids $(n=5)$, functioning endocrine pancreatic tumor (EPTs; $n=8$ ), nonfunctioning EPTs $(n=6)$, appendix carcinoids $(n=6)$, L-cell rectal carcinoids $(n=3)$, serotonin-producing rectal carcinoids $(n=3)$, simple goiter $(n=2)$, medullary thyroid cancer $(n=7)$, adrenocortical adenomas $(n=5)$, adrenocortical cancer $(n=6)$, pheochromocytomas $(n=5)$, neuroblastomas $(n=2)$, follicular thyroid adenomas $(n=5)$, follicular thyroid cancer $(n=2)$, papillary thyroid carcinomas $(n=3)$, and parathyroid adenomas $(n=4)$.

For western blot (WB) analysis, protein was isolated from 21 patients with ileal carcinoids comprising 15 primary tumors, 11 mesenterial metastases, and one liver metastasis. Medical records were reviewed in order to establish the presence of fibrosis-related symptoms present at the time of operation. Intestinal obstruction, ischemia, or abdominal adherences were grouped under the term mesenterial fibrosis. Echocardiograms were performed on all the patients with ileal carcinoids to evaluate the presence of carcinoid heart disease and heart valve stenosis. Ki67 proliferation index was evaluated in all cases by a pathologist and verified by the author J L C. All the patients had metastases at the time of operation.

\section{Immunohistochemistry}

Tumor specimens were fixed in buffered formalin for 1-2 days, dehydrated, and embedded in paraffin wax. Sections, $\sim 4 \mu \mathrm{m}$ thick, were placed on positively charged glass superfrost slides (Menzel-Gläser, Braunschweig, Germany). They were deparaffinized in xylene and rehydrated using decreasing concentrations of ethanol. Antigen retrieval was performed using microwave treatment at $700 \mathrm{~W}$ for $14 \mathrm{~min}$ and $350 \mathrm{~W}$ for $10 \mathrm{~min}$ in Tris $\mathrm{pH} 8$ for CTGF and in citrate buffer $\mathrm{pH} 6$ for $\alpha$-SMA respectively.

To detect CTGF, sections were first incubated for 30 min with $0.1 \% \mathrm{H}_{2} \mathrm{O}_{2}$ to quench endogenous peroxidase, washed with PBS, and then with normal horse serum (Vector Laboratories, CA, USA): redistilled water (1:5), which was tapped off after $30 \mathrm{~min}$ incubation. Sections were then incubated overnight at $4{ }^{\circ} \mathrm{C}$ with polyclonal goat-anti-human CTGF (L: 20 Santa Cruz Biotechnology, Santa Cruz, CA, USA; sc-14939) diluted 1:1000 in PBS with 2\% BSA then with biotinylated anti-goat antibody (Vector BA-9500). Sections were then incubated for $30 \mathrm{~min}$ with avidinbiotin-HRP (Vectastain ABC PK-6100).

Detection of $\alpha$-SMA was performed using EnVision+ System-HRP (DakoCytomation K4011, Glostrup, Denmark) according to the manufacturer's instructions. In short, after a $5 \mathrm{~min}$ incubation with peroxidase block, the slides were then incubated for $1 \mathrm{~h}$ at room temperature with monoclonal mouse anti-human $\alpha$-SMA (DakoCytomation) diluted 1:50 in PBS with 2\% BSA and then with HRP-labeled EnVision polymer for $30 \mathrm{~min}$.

For both CTGF and $\alpha$-SMA detection, sections were washed thrice in PBS pH 7.4 after each step with the exception of after horse serum application. DAB chromogen was applied for $5 \mathrm{~min}$. Counter staining was done using Meyer's hematoxylin, and the sections were then dehydrated with increasing concentrations of ethanol, followed by xylene and then mounted using Pertex mounting medium (Histolab Products AB, Göteborg, Sweden).

The immunostained sections were evaluated in areas with highest number of tumor cells using Zeiss light microscope and graded by independent reviewers (J C, A T, and L G) using the following criteria: nonimmunoreactive $(-) ; 1-25 \%,(+) ; 25-50 \%(++)$; 
and more than 50\% $(+++)$ immunoreactive cells. CTGF IR was examined in tumor cells, while $\alpha$-SMA IR was evaluated in the fibroblasts component of intratumoral stroma. $\alpha$-SMA IR in vascular smooth muscle and capsule when present was not evaluated.

\section{Western blot}

Cytoplasmic extracts were prepared from frozen tissue from 21 patients with ileal carcinoids (15 primary tumors, 11 mesenterial metastases, and 1 liver metastasis) according to the instructions of the NE-PER nuclear and cytoplasmic extraction kit (Pierce Protein Research Products, Thermo Fisher Scientific, Rockford, IL, USA) in the presence of protease inhibitors. The protein content of the cytoplasmic lysates was measured using the BCA Protein assay kit (Pierce). Samples were boiled for 5 min in Laemmli Buffer (Bio-Rad) containing $5 \% \quad \beta$-mercaptoethanol and resolved on $10-20 \%$ Tris-HCl gradient gel (Bio-Rad). Proteins $(40 \mu \mathrm{g})$ were then transferred onto a nitrocellulose membrane and blocked overnight in buffer containing $1 \times$ TBS and $0.1 \%$ Tween 20 with $5 \%$ nonfat dry milk.

Immunodetection was performed by incubating the blots with polyclonal goat-anti-CTGF (L: 20 Santa Cruz Biotechnology sc-14939) diluted 1:1000 in buffer ( $1 \times$ TBS, $0.1 \%$ Tween 20, and 5\% milk) for $3 \mathrm{~h}$ at room temperature. Blots were then washed six times with washing buffer $(1 \times$ TBS and $0.1 \%$ Tween 20$)$ and incubated with secondary HRP-conjugated antibodies (Santa Cruz Biotechnology) for $1 \mathrm{~h}$ at room temperature. After washings, Amersham ECL plus system was used for detection. Prestained molecular weight standards (Dual Color, Bio-Rad) were used to monitor protein migration. Coomassie blue staining was performed on membranes to verify equal sample loading and transfer.

\section{Controls}

To control for non-specific immunostaining, anti-CTGF (L-20) was preincubated with both 10 and $1 \mathrm{nmol} / \mathrm{ml}$ of the respective peptide (Santa Cruz Biotechnology sc-14939P).

\section{Ethics}

The study was reviewed and approved by the local Medical Ethics Committee at Uppsala University Hospital (DiarNr: 2007/006).

\section{Results}

\section{CTGF IR in endocrine tumors}

CTGF expression was detected in all lung carcinoids, medullary thyroid cancer, and gastroenteropancreatic tumors. The number of CTGF-immunoreactive cells, however, varied considerably (see Table 1 and Fig. 1).
Table 1 Immunohistochemical detection of CTGF and $\alpha$-SMA in endocrine tumors.

\begin{tabular}{|c|c|c|c|}
\hline Tumor & Cases & $\begin{array}{c}\text { CTGF } \\
(>50 \%)^{a}\end{array}$ & $\begin{array}{c}\alpha-\text { SMA } \\
(>50 \%)^{b}\end{array}$ \\
\hline \multicolumn{4}{|l|}{ Foregut } \\
\hline ECLoma type 1 & 7 & 0 & 0 \\
\hline ECLoma type 3 & 3 & 0 & 0 \\
\hline Lung carcinoid & 5 & 0 & 0 \\
\hline Endocrine pancreatic tumor & 14 & 2 & 2 \\
\hline \multicolumn{4}{|l|}{ Midgut } \\
\hline Appendix carcinoid & 6 & 0 & 0 \\
\hline Ileal carcinoid & 42 & 42 & 35 \\
\hline \multicolumn{4}{|l|}{ Hindgut } \\
\hline Rectal carcinoid & 6 & 4 & 1 \\
\hline \multicolumn{4}{|l|}{ Other endocrine tumors } \\
\hline Adrenocortical adenoma & 5 & 0 & 0 \\
\hline Adrenocortical cancer & 6 & 0 & 0 \\
\hline Pheochromocytoma & 5 & 0 & 0 \\
\hline Neuroblastoma & 2 & 0 & 0 \\
\hline Follicular thyroid adenoma & 4 & 0 & 0 \\
\hline Follicular thyroid cancer & 2 & 0 & 0 \\
\hline Medullary thyroid cancer & 7 & 0 & 0 \\
\hline Parathyroid adenoma & 4 & 0 & 0 \\
\hline Papillary thyroid cancer & 3 & 0 & 0 \\
\hline
\end{tabular}

${ }^{\mathrm{a}}$ Cases with $>50 \%$ CTGF-immunoreactive tumor cells.

${ }^{\mathrm{b}}$ Cases with $>50 \% \alpha$-SMA-immunoreactive cells in fibroblast component of intratumoral stroma.

Intensive CTGF IR in more than 50\% of tumor cells was detected in all ileal carcinoids. In the other endocrine tumors, comparable relative incidence of CTGF IR was only present in two EPTs, one lung carcinoid and four rectal carcinoids (Table 1). CTGF IR in the remaining tumors ranged from none to $<50 \%$ of tumor cells. CTGF IR was not detected in adrenocortical cancer, pheochromocytomas, neuroblastomas, follicular thyroid adenomas, simple goiters, and parathyroid adenomas. In CTGF-expressing tumors, stroma displayed weak CTGF IR. CTGF IR was strongest in tumor cells adjacent to surrounding fibrovascular stroma.

A correlation was observed between CTGF and serotonin production in endocrine tumors even when ileal carcinoids were excluded from the analysis. This was observed when both absolute presence and absence of CTGF/serotonin IR in tumors (excluding ileal carcinoids) were analyzed $(P<0.05)$ and pronounced when presence and absence $>50 \%$ expression of CTGF/ serotonin were analyzed $(P<0.0001$, Fisher's exact test).

\section{$\alpha-S M A ~ I R$ in endocrine tumors}

$\alpha$-SMA-immunoreactive stromal cells were found in nearly all endocrine tumors studied (see Table 1 and Fig. 1). The highest relative incidence of $\alpha$-SMA IR was present in ileal carcinoids, in which over $83 \%$ of the tumors expressed $\alpha$-SMA in $>50 \%$ of fibroblast-like stromal cells (see Table 1 and Fig. 1). In these tumors, areas of fibrosis were extensive and well vascularized. Comparable $\alpha$-SMA IR was observed in 1 out of 6 rectal carcinoid tumors and 2 out of 14 EPTs (producing 
serotonin and gastrin respectively). Expression of $\alpha$-SMA in more than $25 \%$ of fibroblast-like stromal cells was only noted in tumors where at least more than $25 \%$ of tumor cells were CTGF immunoreactive. $\alpha$-SMA IR was not detected in parathyroid adenomas but was displayed in $<25 \%$ of fibroblast-like stromal cells in appendiceal carcinoids, medullary thyroid cancer, adrenocortical cancer, pheochromocytomas, and follicular thyroid adenomas.

\section{Western immunoblotting of CTGF in ileal carcinoids}

WB of protein extracts from ileal carcinoids using a thrombospondin-1 (TSP-1 or THBS1 as listed in Hugo Database) motif-specific antibody, anti-CTGF L-20,
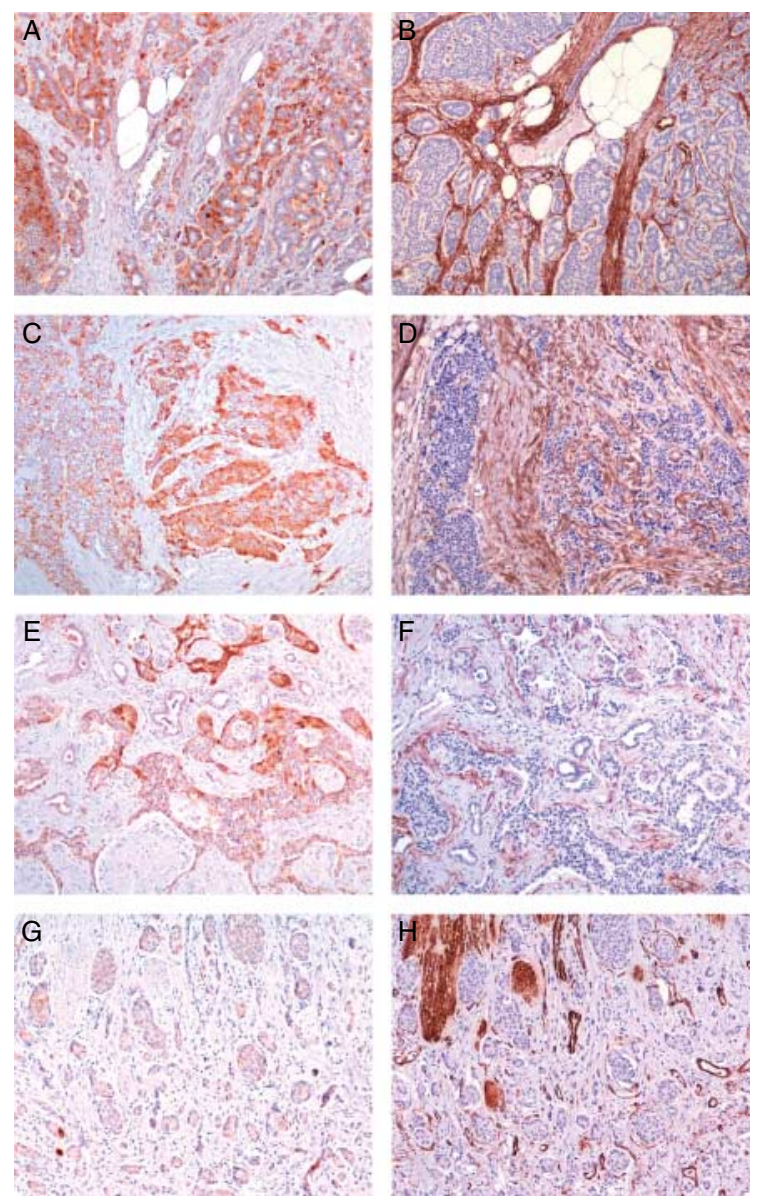

Figure 1 CTGF and $\alpha$-SMA-immunoreactive cells in endocrine tumors. Serial sections from endocrine tumors were used to compare the expression of CTGF and $\alpha$-SMA. In ileal carcinoids, intensive CTGF immunoreactivity (IR) (A and C) was often noted in tumor areas adjacent to stromal areas; $>50 \%$ of fibroblast-like stromal cells were $\alpha$-SMA immunoreactive (B and D). In some EPTs, a high level of CTGF IR was present focally $(E)$. In these areas, a greater number of stromal cells were $\alpha$-SMA immunoreactive $(F)$. Appendiceal carcinoids expressed low levels of CTGF $(\mathrm{G})$, and $\alpha$-SMA-immunoreactive cells were not detected in the stroma surrounding the tumor $(\mathrm{H})$. Original magnification $100 \times$.

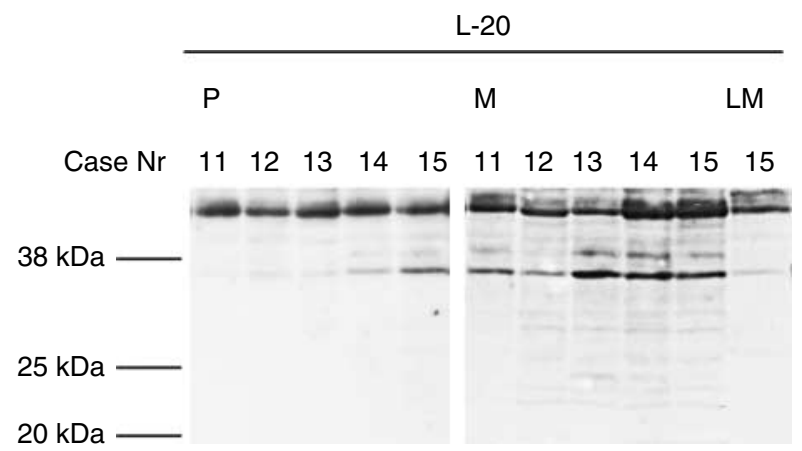

Figure 2 Western blot analysis of CTGF expression in ileal carcinoids: in all ileal carcinoids studied from 21 patients, bands corresponding to full-length CTGF were visualized using western blots immunoblotted with L-20 (TSP-1 motif-specific antibody, anti-CTGF). Lanes $1-5$ contain $f$ protein lysate from five primary tumors $(P)$ where lanes are labeled with patient number. Lanes 6-11 contain protein lysate from mesenterial metastases (M) and liver metastases (LM) from the same patients.

visualized pronounced bands in all the samples that migrated with an apparent molecular weight of 50 and 38-36 kDa (see Fig. 2). A weaker band migrating with an apparent molecular weight of $20 \mathrm{kDa}$ was observed in the majority of tumors. As differences between generally high levels of CTGF in the tumors were subtle, they could not be separated from variations in tumor content. Therefore, no attempt to quantify the relative amounts of CTGF was made. In all 21 cases, local stroma was extensive, and IR of CTGF and $\alpha$-SMA was over 50 and $40 \%$ respectively. Nineteen cases had stage IV, and two had stage IIIb diseases. Fibrosis and adherences surrounding the tumor and in the peritoneal cavity are described in 16 out of 21 patients in the surgical report. In six patients, this fibrosis caused surgically verified intestinal obstruction. Proliferation index, $\mathrm{Ki} 67 \%$, in the ileal carcinoids studied ranged from $<1$ to $8 \%$ (median $<1 \%$ ). Four of twenty-one patients were diagnosed with carcinoid heart disease. These clinicopathological factors, including the presence of distal fibrosis, were not correlated to local CTGF levels, which were generally high.

\section{Controls}

CTGF immunoblotting was completely blocked when anti-CTGF (L-20) was preincubated with $1 \mathrm{nmol}$ of blocking peptide (Santa Cruz Biotechnology sc-14939P or sc-34772P).

\section{Discussion}

CTGF is known to regulate myofibroblast differentiation and collagen production, which are both key mechanisms in fibrosis development. We confirm, in a large series, that ileal carcinoids as a rule show abundant expression of CTGF in both primary tumors 
and metastases. We also show that the majority of fibroblasts-like cells surrounding ileal carcinoid tumor cells express $\alpha$-SMA, indicating myofibroblast differentiation. These results are in agreement with a role for CTGF in ileal carcinoid fibrosis.

In the other endocrine tumors, CTGF IR was less prominent. CTGF IR was found in most gastroenteropancreatic tumors, in medullary thyroid cancer, and in lung carcinoids. Intensive CTGF IR in more than 50\% of tumor cells was noted, apart from ileal carcinoids, in 2 out of 14 EPTs, 1 out of 5 lung carcinoids, and 4 out of 6 rectal carcinoids. CTGF expression in endocrine tumors, apart from ileal carcinoids, is more frequent $(P<0.05)$ but not exclusive to tumors simultaneously producing serotonin. Serotonin-independent mechanisms for CTGF production must, therefore, exist. Serotonin administration induces carcinoid heart disease in rats through a TGFB-mediated mechanism (2), and the presence of serotonin and perhaps other specific hormones likely modulate CTGF function.

CTGF protein bands with an approximate molecular weight of $38,36,23$, and $20 \mathrm{kDa}$ were found in protein lysates from ileal carcinoids. Full-length glycosylated CTGF has an estimated molecular weight of 36-42 kDa. The presence of proteolytic fragments, with an estimated molecular weight of $23 \mathrm{kDa}$, were detected using anti-bodies directed against the C-terminal domains indicating that CTGF is cleaved in the hinge region (19). It has been speculated that CTGF fragments may have specific biological functions (19, 22). High levels of N-terminal CTGF were found in patients with fibrotic scleroderma (23) and in diabetic patients with nephropathy (24). Our preliminary results from WB using anti-N-CTGF (G-14), specific for the N-terminal IGF-binding protein motif, visualized a band migrating at an apparent molecular weight of $50 \mathrm{kDa}$ and, in a subgroup of ileal carcinoids metastases, an additional band at $20 \mathrm{kDa}$. The $20 \mathrm{kDa}$ bands could represent truncated N-terminal CTGF domains, and its presence appears to vary between patients (data not shown). Measurement of circulating N-terminal CTGF levels in plasma and/or in peritoneal fluid in patients with ileal carcinoid may help to understand the relation to extent of fibrosis development seen in individuals.

CTGF is shown to mediate TGFB-induced myofibroblast differentiation $(18,19)$. $\alpha$-SMA-positive fibroblast-like stromal cells were present in all tumors included in our study; however, their prevalence varied. The majority of ileal carcinoids displayed very high proportion of $\alpha$-SMA-immunoreactive fibroblast-like cells in an extensive stroma. The term 'fibroblast-like' is used to guard for potential error resulting from a-SMA expression in lengthwise-sectioned smooth muscle cells surrounding stromal vascular structures, which can be difficult to distinguish from fibroblasts in some cases. Differentiated $\alpha$-SMA expressing myofibroblasts produce collagen, whereas proliferating fibroblasts neither express $\alpha$-SMA nor produce collagen (18).
Our results indicate that it is likely that myofibroblasts contribute to the excessive local fibrosis that is often present in ileal carcinoids and CTGF may be involved in mediating this process. Myofibroblasts in these tumors may also be involved in tumor vascularization, invasiveness, and early metastases formation, which are also features of ileal carcinoids (25-27). This potential mechanism may not be unique for ileal carcinoids as it can be noted that stromal $\alpha$-SMA IR was relatively high $(>25 \%)$ in the other endocrine tumors that displayed CTGF IR in $>50 \%$ of tumor cells. Both EPTs (producing serotonin and gastrin respectively) and the lung carcinoid had formed metastases, while only one of the four rectal carcinoids had formed lymph node metastases.

A multifunctional role for CTGF in tumorigenesis is emerging. CTGF is expressed at high levels in other human cancers and is reported to correlate with both tumor stage and survival. The results, however, are contradictory. CTGF is associated with increased proliferation and bad prognosis in glioblastomas (28), while in colorectal cancer and non-small cell lung cancer, high CTGF expression is associated with improved survival $(29,30)$. Likewise, CTGF is thought to contribute to tumor invasiveness in glioblastomas (31) and in osteolytic breast cancer (32), while overexpression of CTGF inhibits metastases and invasion of human lung cancer cells (29), and attenuates the growth and tumorigenicity of squamous cell carcinoma cells (33). The biology behind the different roles has not been explained, but it is becoming apparent that other signaling molecules act in concert with CTGF in combinatory signal pathways with different outcomes (18). It seems, however, that CTGF may play a role in the development of certain tumors and could be a target for future therapy. A study reports that a human CTGF-specific MAB deceases tumor growth and metastases, and attenuates tumor angiogenesis and cancer cell proliferation in an orthotopic mouse model of pancreatic cancer (34).

The role of CTGF in ileal carcinoids is unknown. CTGF has been shown to stimulate proliferation of cells isolated from a ECL cell tumor but not of ECL cells isolated from normal mucosa (35). Ileal carcinoids display generally low proliferation index (36), and CTGF expression was not associated with tumor proliferation index in this study. A potential proliferative role for CTGF in these tumors is, therefore, likely dependent on the presence of other factors.

CTGF expression is generally high in ileal carcinoids especially in tumor areas adjacent to extensive $\alpha$-SMAexpressing stroma. This supports a role for CTGF in ileal carcinoid fibrosis. CTGF is also expressed in other gastroenteropancreatic and lung endocrine tumors, however, most often at lower relative incidence. Complications due to fibrosis are a major concern for patients with ileal carcinoids, and CTGF may provide a target for future treatment. 


\section{Declaration of interest}

The authors declare that there is no conflict of interest that could be perceived as prejudicing the impartiality of the research reported.

\section{Funding}

The Swedish Cancer Society (project number 4880-B05-03XBB), The Lions Fund for Cancer Research at Uppsala University Hospital, and The Erik, Karin, and Gösta Selanders Fund are acknowledged for providing financial support for this study.

\section{Acknowledgements}

The authors thank Åsa Forsberg for her excellent technical assistance, Prof. Lars Grimelius for help in gathering and in evaluating the tumor material.

\section{References}

1 Modlin IM, Oberg K, Chung DC, Jensen RT, de Herder WW, Thakker RV, Caplin M, Delle Fave G, Kaltsas GA, Krenning EP, Moss SF, Nilsson O, Rindi G, Salazar R, Ruszniewski P \& Sundin A. Gastroenteropancreatic neuroendocrine tumours. Lancet Oncology 20089 61-72. (doi:10.1016/S1470-2045(07)70410-2)

2 Gustafsson BI, Tommeras K, Nordrum I, Loennechen JP, Brunsvik A, Solligard E, Fossmark R, Bakke I, Syversen U \& Waldum H. Long-term serotonin administration induces heart valve disease in rats. Circulation 2005111 1517-1522. (doi:10. 1161/01.CIR.0000159356.42064.48)

3 Rameshwar P, Oh HS, Yook C, Gascon P \& Chang VT. Substance p-fibronectin-cytokine interactions in myeloproliferative disorders with bone marrow fibrosis. Acta Haematologica 2003109 1-10. (doi:10.1159/000067268)

4 Nilsson J, von Euler AM \& Dalsgaard CJ. Stimulation of connective tissue cell growth by substance P and substance K. Nature 1985 315 61-63. (doi:10.1038/315061a0)

5 Katayama I \& Nishioka K. Substance P augments fibrogenic cytokine-induced fibroblast proliferation: possible involvement of neuropeptide in tissue fibrosis. Journal of Dermatological Science 199715 201-206. (doi:10.1016/S0923-1811(97)00608-7)

6 Modlin IM, Shapiro MD \& Kidd M. Carcinoid tumors and fibrosis: an association with no explanation. American Journal of Gastroenterology 200499 2466-2478. (doi:10.1111/j.15720241.2004.40507.x)

7 Wulbrand U, Wied M, Zofel P, Goke B, Arnold R \& Fehmann H. Growth factor receptor expression in human gastroenteropancreatic neuroendocrine tumours. European Journal of Clinical Investigation 199828 1038-1049. (doi:10.1046/j.1365-2362. 1998.00397.x)

8 Nilsson O, Wangberg B, McRae A, Dahlstrom A \& Ahlman H. Growth factors and carcinoid tumours. Acta Oncologica 199332 115-124. (doi:10.3109/02841869309083899)

9 Zhang PJ, Furth EE, Cai X, Goldblum JR, Pasha TL \& Min KW. The role of $\beta$-catenin, TGF $\beta 3$, NGF2, FGF2, IGFR 2 , and BMP4 in the pathogenesis of mesenteric sclerosis and angiopathy in midgut carcinoids. Human Pathology 200435 670-674. (doi:10.1016/j.humpath.2003.12.010)

10 Grotendorst GR, Okochi H \& Hayashi N. A novel transforming growth factor beta response element controls the expression of the connective tissue growth factor gene. Cell Growth $\mathcal{E}$ Differentiation $19967469-480$.

11 Duncan MR, Frazier KS, Abramson S, Williams S, Klapper H, Huang X \& Grotendorst GR. Connective tissue growth factor mediates transforming growth factor beta-induced collagen synthesis: down-regulation by cAMP. FASEB Journal 199913 1774-1786.
12 Weston BS, Wahab NA \& Mason RM. CTGF mediates TGF-betainduced fibronectin matrix deposition by upregulating active alpha5beta1 integrin in human mesangial cells. Journal of the American Society of Nephrology 200314 601-610. (doi:10.1097/ 01.ASN.0000051600.53134.B9)

13 Rachfal AW \& Brigstock DR. Connective tissue growth factor (CTGF/CCN2) in hepatic fibrosis. Hepatology Research 200326 1-9. (doi:10.1016/S1386-6346(03)00115-3)

14 Kidd M, Modlin IM, Shapiro MD, Camp RL, Mane SM, Usinger W \& Murren JR. CTGF, intestinal stellate cells and carcinoid fibrogenesis. World Journal of Gastroenterology 200713 5208-5216.

15 Mauviel A. Transforming growth factor-beta: a key mediator of fibrosis. Methods in Molecular Medicine 2005117 69-80. (doi:10.1385/1-59259-940-0:069)

16 Leask A \& Abraham DJ. TGF-beta signaling and the fibrotic response. FASEB Journal 200418 816-827. (doi:10.1096/fj.031273rev)

17 Chaudhry A, Oberg K, Gobl A, Heldin CH \& Funa K. Expression of transforming growth factors beta 1 , beta 2 , beta 3 in neuroendocrine tumors of the digestive system. Anticancer Research 199414 2085-2091.

18 Grotendorst GR, Rahmanie H \& Duncan MR. Combinatorial signaling pathways determine fibroblast proliferation and myofibroblast differentiation. FASEB Journal $2004 \quad 18$ 469-479. (doi:10.1096/fj.03-0699com)

19 Grotendorst GR \& Duncan MR. Individual domains of connective tissue growth factor regulate fibroblast proliferation and myofibroblast differentiation. FASEB Journal 200519 729-738. (doi:10.1096/fj.04-3217com)

20 Solcia E, Klöppel G \& Sobin LH. Histological Typing of Endocrine Tumours. WHO International Histological Classification of Tumours. Berlin: Springer, 2000.

21 Kloppel G, Couvelard A, Perren A, Komminoth P, McNicol AM, Nilsson O, Scarpa A, Scoazec JY, Wiedenmann B, Papotti M, Rindi G \& Plockinger U. ENETS Consensus Guidelines for the Standards of Care in Neuroendocrine Tumors: towards a standardized approach to the diagnosis of gastroenteropancreatic neuroendocrine tumors and their prognostic stratification. Neuroendocrinology $2009 \quad 90 \quad 162-166 . \quad$ (doi:10.1159/ 000182196)

22 Yang DH, Kim HS, Wilson EM, Rosenfeld RG \& Oh Y. Identification of glycosylated $38-\mathrm{kDa}$ connective tissue growth factor (IGFBPrelated protein 2) and proteolytic fragments in human biological fluids, and up-regulation of IGFBP-rP2 expression by TGF-beta in Hs578T human breast cancer cells. Journal of Clinical Endocrinology and Metabolism 199883 2593-2596. (doi:10.1210/jc. 83.7.2593)

23 Dziadzio M, Usinger W, Leask A, Abraham D, Black CM, Denton C \& Stratton R. N-terminal connective tissue growth factor is a marker of the fibrotic phenotype in scleroderma. QJM: Monthly Journal of the Association of Physicians 200598 485-492. (doi:10. 1093/qjmed/hci078)

24 Roestenberg P, van Nieuwenhoven FA, Wieten L, Boer P, Diekman T, Tiller AM, Wiersinga WM, Oliver N, Usinger W, Weitz S, Schlingemann RO \& Goldschmeding R. Connective tissue growth factor is increased in plasma of type 1 diabetic patients with nephropathy. Diabetes Care 200427 1164-1170. (doi:10. 2337/diacare.27.5.1164)

25 Nielsen BS, Rank F, Illemann M, Lund LR \& Dano K. Stromal cells associated with early invasive foci in human mammary ductal carcinoma in situ coexpress urokinase and urokinase receptor. International Journal of Cancer 2007120 2086-2095. (doi:10. 1002/ijc.22340)

26 De Wever O \& Mareel M. Role of tissue stroma in cancer cell invasion. Journal of Pathology 2003200 429-447. (doi:10.1002/path.1398)

27 Desmouliere A, Guyot C \& Gabbiani G. The stroma reaction myofibroblast: a key player in the control of tumor cell behavior. International Journal of Developmental Biology $2004 \mathbf{4 8} 509-517$. (doi:10.1387/ijdb.041802ad)

28 Xie D, Yin D, Wang HJ, Liu GT, Elashoff R, Black K \& Koeffler HP. Levels of expression of CYR61 and CTGF are prognostic for tumor 
progression and survival of individuals with gliomas. Clinical Cancer Research 200410 2072-2081. (doi:10.1158/1078-0432. CCR-0659-03)

29 Chang CC, Shih JY, Jeng YM, Su JL, Lin BZ, Chen ST, Chau YP, Yang PC \& Kuo ML. Connective tissue growth factor and its role in lung adenocarcinoma invasion and metastasis. Journal of the National Cancer Institute 200496 364-375. (doi:10.1093/jnci/ djh059)

30 Lin BR, Chang CC, Che TF, Chen ST, Chen RJ, Yang CY, Jeng YM, Liang JT, Lee PH, Chang KJ, Chau YP \& Kuo ML. Connective tissue growth factor inhibits metastasis and acts as an independent prognostic marker in colorectal cancer. Gastroenterology 2005 128 9-23. (doi:10.1053/j.gastro.2004.10.007)

31 Pan LH, Beppu T, Kurose A, Yamauchi K, Sugawara A, Suzuki M, Ogawa A \& Sawai T. Neoplastic cells and proliferating endothelial cells express connective tissue growth factor (CTGF) in glioblastoma. Neurological Research 200224 677-683. (doi:10.1179/ $016164102101200573)$

32 Xie D, Nakachi K, Wang H, Elashoff R \& Koeffler HP. Elevated levels of connective tissue growth factor, WISP-1, and CYR61 in primary breast cancers associated with more advanced features. Cancer Research $2001618917-8923$.

33 Moritani NH, Kubota S, Nishida T, Kawaki H, Kondo S, Sugahara T \& Takigawa M. Suppressive effect of overexpressed connective tissue growth factor on tumor cell growth in a human oral squamous cell carcinoma-derived cell line. Cancer Letters 2003 192 205-214. (doi:10.1016/S0304-3835(02)00718-8)

34 Brigstock DR, Steffen CL, Kim GY, Vegunta RK, Diehl JR \& Harding PA. Purification and characterization of novel heparinbinding growth factors in uterine secretory fluids. Identification as heparin-regulated $\mathrm{Mr} 10,000$ forms of connective tissue growth factor. Journal of Biological Chemistry 1997272 20275-20282. (doi:10.1074/jbc.272.32.20275)

35 Kidd M, Modlin IM, Eick GN, Camp RL \& Mane SM. Role of CCN2/CTGF in the proliferation of Mastomys enterochromaffinlike cells and gastric carcinoid development. American Journal of Physiology. Gastrointestinal and Liver Physiology 2007 292 G191-G200. (doi:10.1152/ajpgi.00131.2006)

36 Cunningham JL, Grimelius L, Sundin A, Agarwal S \& Janson ET. Malignant ileocaecal serotonin-producing carcinoid tumours: the presence of a solid growth pattern and/or Ki67 index above 1\% identifies patients with a poorer prognosis. Acta Oncologica 2007 46 747-756. (doi:10.1080/02841860701218659)

Received 6 July 2010

Accepted 26 July 2010 